\title{
PHASE TRANSITIONS IN COULOMB SYSTEMS
}

\author{
J.P. Hansen \\ Laboratoire de Physique Théomique des Liquides», Université P. et M. Curie, \\ 75230 Paris Cedex 05, France
}

Résumé - On donne une brève revue de certains résultats théoriques concernant les transitions de phase dans des modèles simples de systèmes coulombiens simples. On met 1'accent sur l'application éventuelle de ces résultats à des suspensions de colloîdes chargês. L'application de la mécanique statistique à une telle suspension révèle l'existence possible d'une transition réversible "liquide-gaz" distincte de la coagulation irréversible.

\begin{abstract}
A brief review is given of some theoretical results on phase transitions in simple models of classical Coulomb systems. The possible relevance of these results for charged colloidal suspensions is pointed out. A Statistical Mechanics treatment of a suspension of charged colloidal particles indicates the possible existence of a reversible "liquid-gas" transition distinct from irreversible coagulation, under favourable physica1 conditions.
\end{abstract}

\section{INTRODUCTION}

Solid colloidal particles suspended in water are frequently highly charged, due to the dissociation of surface radicals in the highly polar solvent. Consequently Coulomb forces play an important role in determining the stability, the structure and the thermodynamics of such suspensions. The Statistical Mechanics of Coulomb systems is presently a very active area of research and discovery, and has recently been the object of another Les Houches workshop(1). In this review an attempt will be made to show how some of the results obtained for simple models of classical Coulomb fluids might be relevant for the study of charged colloids. The emphasis will be on phase transitions in such models, including melting, condensation and ion pairing ("conductor-insulator" transition), both in three and in two dimensions. In the second part of this paper, the possible existence of a reversible "liquid-gas" transition in charged colloidal suspensions, distinct from irreversible coagulation, will be examined. It will be shown that such a transition should be expected in a relatively narrow range of stray salt concentrations and for particles typically one micron in diameter.

\section{SINGLE COMPONENT MODELS}

The familiar DLvo potention between the double ${ }_{3}$ fayers surrounding spherical particles of diameter $\sigma$ is of the form $(2,3)$ :

$$
v(r)=\gamma^{2} \frac{e^{-k(n-\sigma)}}{r}
$$

\footnotetext{
* Equipe associée au C.N.R.S.
} 
where $\mathbf{r}$ is the distance between the centers of the spheres, $\mathrm{K}^{-1}$ is the Debye screening length and

$$
\begin{aligned}
\gamma^{2} & =16 \pi \varepsilon_{0} \varepsilon \sigma^{2}\left(\frac{-k_{B} T}{e}\right)^{2} \tanh ^{2}\left(\frac{e \psi_{0}}{4 k_{B} T}\right) \\
& \cong \pi \varepsilon_{0} \varepsilon \sigma^{2} \psi_{0}^{2}
\end{aligned}
$$

where the second line holds if the surface potential $\psi_{0}$ of the colloidal particles is less than $\mathrm{k}_{\mathrm{B}} \mathrm{T} / \mathrm{e}$ (the counter-ions and salt ions are assumed to be monovalent). In the weak screening (1inear) regime, $\psi_{0}$ is proportional to the total surface charge Ze carried by the macroions, so that $v(r)$ takes the form of a screened Coulomb potentia1:

$$
v(r)=\frac{z^{2} e^{2}}{\varepsilon r} e^{-k(r-\sigma)}
$$

where $\mathrm{e}$ is expressed in electrostatic units. For very weak screening $(\mathrm{K} \rightarrow 0)$, a regime corresponding typically to the absence of added salt, the colloidal particles interact via the bare coulomb potential and move in a practically uniform neutralizing background of counterions. Neglecting steric repulsion for the moment, which amounts to setting

$\sigma=0$ (this point will be reexamined later), the colloidal system is then reasonably well described by the "one component plasma" (OCP) model of $\mathrm{N}$ point charges immersed in a uniform background of opposite charge. The total potential energy $V_{N}$ for this model includes particle-particle, particle-background and background-background coulomb terms. For a periodic system in a volume $\Omega, V_{N}$ is most compactly expressed in terms of the Fourier components of the microscopic density:

$$
\begin{aligned}
& P_{k}=\sum_{i} e^{i \vec{k} \cdot \vec{i}} \\
& V_{N}=\frac{1}{\Omega} \sum_{k}^{\prime} \hat{v}(k)\left[\rho_{k} \rho-k-N\right]
\end{aligned}
$$

where the primed sum runs over all wave-numbers $\vec{k} \neq$ o compatible with the periodic boundary conditions, the $\vec{k}=\vec{o}$ term being left out to account for the background interactions (electroneutrality); $\hat{v}(k)$ is the Fourier transform of the Coulomb potential

$$
\hat{v}(k)=\frac{4 \pi z^{2} e^{2}}{\varepsilon k^{2}}
$$

The OCP is the simplestmodel of a Coulomb system, and it is the classical equivalent of Wigner's "jellium" model for the electron gas (where $Z=-1$ and $\varepsilon=1$ ). In view of its simplicity it is one of the most widely studied and best understood models of Statistical Mechanics $(4)$. An attractive feature of the model, which follows immediate$1 y$ from inspection of its partition function and the scale invariance of the purely Coulombic potential $(v(r / \lambda)=\lambda v(r))$, is that its excess (non-ideal) properties are functions of the single dimensionless parameter $\Gamma$, the ratio of the Bjerrum (or Landau) length $b$ over the ion-sphere radius a:

$$
\begin{gathered}
\Gamma=\frac{b}{a}=\frac{z^{2} e^{2}}{\varepsilon a k_{B} T} \\
a=(3 \Omega / 4 \pi N)^{1 / 3}=(3 / 4 \pi \rho)^{1 / 3}
\end{gathered}
$$

In the strong coupling limit $\Gamma \gg 1$, the thermodynamic properties of the model are given quite accurately (within a few percent) by the 
simple ion-sphere model, in which each ion is confined to a sphere of radius a and interacts only with the uniform background contained in that sphere, which exactly cancels the ionic charge. An elementary electrostatic calculation shows that the ion is subject to a harmonic potential; the reduced excess internal energy per ion is then given by:

$$
u=\frac{u^{\text {ex }}}{N k_{B} T}=\frac{9}{10} \Gamma+\frac{3}{2}
$$

where the first term is purely static (ion at the center of its sphere) and the second term is the thermal energy (equipartition theorem). In the strong coupling limit, the pair distribution function $g(r)$ as calculated from "exact" simulations or from approximate integral equations (of which the HNC equation is by far the most accurate for this model, (4) exhibits both a wel1-defined "correlation hole" ( $g(r)=$ o for $r \leqslant a)$ and pronounced oscillations (short range order). The resulting static

$S(k)=\frac{1}{N}\left\langle\rho_{k} \rho-k\right\rangle=1+4 \pi \rho \int_{0}^{\infty} \frac{\sin k r}{k r}[g(r)-1] r^{2} d r$

is very liquid-like, except at smalf wavenumbers, where it vanishes like $\mathrm{k}^{2}$, due to perfect screening ${ }^{2}$, instead of tending to the isothermal compressibility. A very characteristic feature of the strongly coupled OCP $(\Gamma \geq 130)$ (16) that the position of the main peak in $S(k)$, $k_{\max }$, scales like $\tilde{\rho}^{19 \zeta(16)}$, in other words:

$$
\mathrm{k}_{\max } \mathrm{a}=\text { constant } \simeq 4.2
$$

such a scaling behaviour is precisely observed in $S(k)$ of dispersions of chargef polystyrene spheres as measured by light scattering experiments 6,7$\}$. In fact the theoretical structure factors of the OCP model have been used to analyse the light scattering data. in the weak screening regime ${ }^{(8)}$.

It is interesting to note in passing that the OCP model is also an excellent starting point for, the analysis of neutron or X-ray scattering data from liquid alkali(lo, fI), where the scaling relation (9) is also well obeyed.

For sufficiently strong coupling, the OCP undergoes a first order transition from a fluid to a crystaline BCC phase ${ }^{9}$. The precise 10cation of the transition is a very delicate task, since the free energies of both phases, as determined by computer simulations, 1 ie very close over an extensive range of $\Gamma$ values. This point can be understood from eq. (7) for the internal energy: the thermal part (which is just $3 / 2$ in the simple ion sphere mode1) which essentially distinguishes both phases, is only a small fraction of the total when $\Gamma>100$. The latest results obtained from very long runs on the Los Alamos computers and a careful analysis of the $\mathrm{N}$-dependence ff the simulated samples, lead to the estimate $\Gamma_{m} \leftrightarrows 178$ at melting $\leftrightarrows 2 f$. Remembering eqs. (6)this leads to the following form of the melting curve:

$$
T_{m}(K)=1.51 \times 10^{-5} \frac{z^{2}}{\varepsilon}\left[\rho_{m}\left(\mathrm{~cm}^{-3}\right)\right]^{1 / 3}
$$

The entropy change per particle at melting, $\Delta S / N k_{B} \cong 0.82^{(9)}$ turns out to be close to that of the hard sphere model, white 3 he volume change of the OCP at melting is, strictly speaking, zeroli3), compared to a $10 \%$ increase in the hard sphere case.

The OCP model can be accommodated to account for the finite size ( $\sigma \neq 0$ ) of the colloidal particles. The presence of an extended correlation hole in the strong coupling regime means that contact configurations 
between the particles will be effectively prevented by the Coulomb repulsion as long as $\sigma<a$, if $\Gamma \gg 1$. In other words it is expected that the structural and thermodynamic properties of a model of equaliy charged hard spheres in a neutralizing background will be identical to those of the OCP if the preceding condition is fulfilled. This observation is in fact the basis of a very successful theory of the $O C P$ structure based on an analytic solution of the "mean spherical approximation" (MSA) for the charged hard sphere model (14). Another consequence of this observation is that the measured structure factor will be completely insensitive to the precise shape and polydispersity of the colloidal particles, as long as the Coulomb repulsion dominates the short-range steric effects. Finally a distinction must be made between the cases where the neutralizing background permeates the colloidal spheres, or, on the contrary, is excluded from the volume of the spheres. The latter situation is of course the physically relevant one, whereas the former situation is more convenient for theoretical analysis. Fortunately both cases can be shown to be equivalent, except for a rather trivial renormalization of the charge: the fraction of the background inside the spheres reduces the apparent charges of these spheres, so that according to Newton's theorem a system of hard spheres of charge $\mathrm{Ze} / 1-\eta$ ) (where $\eta=\Pi \rho \sigma^{3} / 6$ is the packing fraction) in a penetrating background is expected to be equivalent to a system of hard spheres of reduced charge $\mathrm{ze}$ immersed in an excluded background $(16)$. The melting transition will be shifted accordingly.

With increasing salt concentration, screening effects become more and more important, and the bare Coulomb potential between colloidal particles must be replaced by a screened potential of the form (3). As Iong as $\mathrm{K} \sigma \ll<1$, the difference between screened and bare Coulomb potentials can be looked upon as a perturbation, the OCP being considered as a zeroth order "reference system". Neglecting again core effects which are negligible for sufficiently strong Coulomb couplings, the structure factor $S(k)$ of the screened system can be related to that of the OCP
(RPA)
$(15,8)$

$$
S(k)=\frac{S_{0}(k)}{1+\rho \beta \hat{v}(k) S_{0}(k)}
$$

where $\hat{w}(k)$ is the "perturbation", i.e. the difference between screened and bare Coulomb potentials:

$$
\hat{n}(k)=-\frac{4 \pi Z^{2} e^{2} K^{2}}{\varepsilon k^{2}\left(k^{2}+K^{2}\right)}
$$

The exact small $\mathrm{k}$ expansion of the OCP structure factor reads (4)

$$
S(k)=\frac{k^{2}}{K_{0}^{2}}\left[1-\delta \frac{k^{2}}{K_{0}^{2}}\right]
$$

where $K_{0}$ is the inverse Debye screening length for the bare OCP(i.e. without counter-ion or salt contributions) and $\delta^{-1}=\beta \rho \chi_{\text {To }}$ is the reduced isothermal compressibility of the OCP (which becomes in fact negative for $\Gamma>3$ ). Combination of eqs.11-13 leads immediately to the $\mathrm{k} \rightarrow \mathrm{o}$ limit and hence to the reduced osmotic compressibility) 
of the screened system(21)

$$
\lim _{k \rightarrow 0} S(k)=\beta \rho X_{T}=\frac{K^{2}}{K_{0}^{2}\left[1-\frac{K^{2}}{K_{0}^{2}}\right]}
$$

Switching from surface charge to surface potential (cf.eqs.1-3), this relation can in principle be used to estimate the surface potential $\Psi_{0}$ from a measurement of $S(k=0)(8)$.

An alternative, variational approach for the calculation of the thermodynamic properties is based on the Gibb-Bogoliubov inequality for the free energies of the screened system (F) and of the reference OCP sys$\operatorname{tem}\left(\mathrm{F}_{0}\right)$ :

$$
F \leqslant F_{0}+\left\langle W_{N}\right\rangle_{0}
$$

where the second term is the average of the total perturbation:

$$
W_{N}=V_{N}-V_{N}^{(0)}=\sum_{i<} \sum_{j} N\left(r_{i j}\right)
$$

calculated over the reference system ensemble. The r.h.s. of eq. (15) can be minimized with resnect to a variational parameter, e.g. an effective valence $z$ effof the reference system ${ }^{(15)}$. The corresponding effective coupling, $f_{T}$, will be lower than the $\Gamma$ calculated from the true valence because the screening can only lead to a decrease of the effective charge. This approach has been applied with success to the
alkali metals (where the screening is due to the conduction electrons), but has apparently not yet been tried for charged colloidal systems. A "rule of the thumb" would state that melting of a screened colloidal crystal will occur whenever $\Gamma$, as determined by minimizing eq. (13), reaches its OCP value $\Gamma_{m}=178$.

When the screening becomes very strong $(K \sigma \gg>1)$, it is expected that the OCP model ceases to be a useful reference system, because the perturbation (12) becomes too large. The hard sphere model, with an effective diameter $\sigma$ eff $>\sigma$, will then be the more useful reference system. This optimum diameter $\sigma$ effan also be determined by a variational calculation based on the inequality (15), or by other standard perturbation theories of the theory of liquids $(18)$; an illustration of such a procedure will be given in the last section of this paper. In the strong screening regime, which corresponds to high concentrations of added salt (but below the coagulation threshold), crystallization is expected to occur whenever the effective paoking fraction $\eta_{\mathrm{eff}}=$ $\pi \rho \sigma^{3}$ eff $/ 6$ reaches the critical value for the hard sphere transition, i.e. $\mathrm{n}=0.5$.

In the difficult intermediate screening regime (Ko 1), neither reference system is truly satisfactory, and one must rely on computer simulations (19) or on specially adapted theories, like the "rescaled" mean spherical approximation (MSA) $(20)$ to predict accurate structure factors or thermodynamic properties. No simple predictions for the melting curve are available in that case.

\section{ONE COMPONENT COULOMB SYSTEMS IN TWO DIMENSIONS}

Colloidal suspension in restricted (slab) geometries, or at the 1 iquidair interface, form fascinating two-dimensional systems, which have attracted a keen interest of the experimentalists(22). For that reason it is of interest to review briefly our present knowledge on phase transitions in two-dimensional (2d) Coulomb systems. Like the preceding section, the present one is restricted to one-component systems. 
One must carefully distinguish between $2 \mathrm{~d}$ systems of particles interacting via the $3 \mathrm{~d}$ Coulomb potential $(1 / \mathrm{r})$ and truly $2 \mathrm{~d}$ systems, where the Coulomb potential, i.e. the solution of the $2 \mathrm{~d}$ Poisson equation, is logarithmic.

a) $1 / \mathrm{r}$ potential

This is the physically relevant potential for layers of spherical colloidal particles. There is an interesting analogy with two-dimensional electron layers trapped at the surface of liquid Helium, which have been extensively studied at Be1l Laboratories (23) and at Saclay(24). In these layers, the electrons crystallize into a 2 d hexagonal lattice although they stay far apart (the lattice spacing is typically 1 micron); but due to the low temperature ( $\mathrm{T} \cong 1 \mathrm{~K}$ ), the coupling constant $\Gamma$ defined in eq. (6) (with $a=(1 / \pi \rho)^{1 / 2}$ in $2 d$. $\rho$ being the areal density) is typicaliy larger than 100 ! In fact experiments (23) and computer simulations(25) agree on the value $\Gamma_{\mathrm{m}} \simeq 130$ at melting. The transition appears to be first order as in the case of $2 \mathrm{~d}$ systems of hard core particles $(26)$, but attempts have been made to use the dislocation-unbinding mechanism $(27)$ to study the melting of the $2 \mathrm{~d}$ electron lattice(28). It is important to stress that the electron layers obey essentially classical Statistical Mechanics, despite the low temperature, because their spacing is much larger than their thermal de Broglie wavelength. The analogy with charged colloidal spheres constrained to $2 \mathrm{~d}$ is hence complete, provided the screening is sufficiently weak so that Coulomb correlations mask any steric effects. The thermal factor $\varepsilon k_{B} T$ for colloidal suspensions is typically 20000 times larger than in the case of electron layers, but this is well compensated by the valence factor $Z^{2}$, so that comparable values of $\Gamma$ are recovered. In the strong screening regime, the hard disk system becomes the relevant model for the study of the $2 \mathrm{~d}$ melting transition. A sizeable volume change on melting (of the order of $10 \%$ ) should be observed in the latter case $(26)$, while this volume change is expected to drop to zero when the salt concentration, and hence the screening, decreases. But the entropy of melting remains finite in the weak screening limit, being of the order of $\Delta S / N k_{B}=0.3(25)$.

Polystyrene spheres trapped at the water-air interface carry large dipole moments $\overrightarrow{\vec{p}}$ perpendicular to. the interfacial plane, due to the asymmetric of the charge distribution around the partly immersed particles.

The parallel dipoles repel each other via the dipolar potential:

$$
v(r)=\frac{\mu^{2}}{r^{3}}
$$

The corresponding dimensionless coupling constant is now

$$
\Gamma=\frac{\mu^{2}}{a^{3} k_{B} T}
$$

For sufficiently large values of $\Gamma$, the interfacial colloid is expected to crystallize. An interfacial hexagonal crystal has indeed been observed experimentally (29) and simulations lead to the estimate $\Gamma_{m} \simeq 60$ at melting; the corresponding entropy change per particle is very similar to that esfimated for the interfacial Coulomb crystal $(\Delta \mathrm{S} / \mathrm{Nk} \backsim 0.3(30)$. It should be stressed however that a direct comparison between theory and experiment is not possible, since the simulations assume the point dipole interaction (17) while the true interaction between extended charge distributions is certainly more complicated. 
b) $\log r$ interaction

The solution of the $2 \mathrm{~d}$ Poisson equation is potential:

$$
v(r)=-z^{2} e^{2} \ln (\Omega / L)
$$

where $L$ is an arbitrary length scale.(19) is precisely the interaction between infinitely long parallel rods carrying a linear charge density Ze. The two-dimensional OCP model of point charged interacting through the potential (19) may hence be a reasonable approximation for the motion of parallel charged rods (like the Tobacco Mosaic Virus) in the plane perpendicular to their axis, provided that the screening length of the counter-ions and the stray ions is significantly larger than the

spacing between the rods.

is independent of density, and a simple scaling argument shows that the equation-of-state is rigorously given at all temperatures by (3):

$$
\frac{\beta P}{\rho}=1-\frac{z^{2} e^{2}}{4 k_{B} T}=1-\frac{\Gamma}{4}
$$

Computer "experiments" have shown that the model undergoes a first order transition from a fluid phase to a hexagonal crystal when Pي140(32, 33). The "molecular dynamics" simulations of Choquard and Clérouin (34) have revealed very interesting cooperative premelting motions of groups of particles, just below the melting temperature; these motions include cyclic permutations of 6,8 or more particles, similar to premelting motions observed for the hard disk fluid, pointing to some kind of universal melting behaviour in 2d. This "Universality" is confirmed on the fluid side of the transition by the striking similarity of the main peak of the structure factors $S(k)$ in these radically different systems 32 )

\section{TWO COMPONENT COULOMB SYSTEMS}

Up to now the discussion has been restricted to one component bare or screened Coulomb system. In such models the counterions and stray ions are represented by a continuum which is characterized only by its screening length $\mathrm{K}^{-1}$. This picture is reasonable whenever the macroions are much larger than the other ions, and it is the basis of the standard DLVO potential between colloidal particles. This and the next section are devoted to two- component Coulomb systems made up of oppositely charged ions of comparable size. The discussion will be restricted to the so-called "primitive model" of electrolytes in which the ions are modeled by charged hard spheres (hard disks in $2 \mathrm{~d}$ ). The shortrange steric repulsion between oppositely charged ions is essential to guarantee stability of classical systems against Coulomb collapse in $3 \mathrm{~d}$. The model has been extensively used to study ionic solutions and molten salts (alkali halides), particularly in its "restricted" version (RPM) where the diameters and absolute charges of all ions are equa1, so that:

$$
\begin{aligned}
v_{\alpha \beta}(r) & =\infty ; r<\sigma \\
& =\frac{z_{\alpha} z_{\beta} e^{2}}{\varepsilon r} ; r>\sigma\left(z_{\alpha}, z_{\beta}= \pm 1\right)
\end{aligned}
$$


A thermodynamic state of this system is characterized by two variables chosen to be the coupling $\Gamma$ in eq. (6), or equivalently

$$
\gamma=\frac{f}{\sigma}=\frac{e^{2}}{\varepsilon \sigma k_{B} T}
$$

and the packing fraction $n=\pi \rho \sigma^{3} / 6$, where $\rho$ is the total number of ions per unit volume. The RPM is a reasonable model for the study of the structure of molten alkali halides, but it is much less satisfactory for ionic solutions because ion-solvent effects are completely ignored; this is not justified when ions and solvent molecules are of comparable size.

Molten salts undergo a liquid-solid transition for couplings $\Gamma \bumpeq 60$, which is accompanied by an unusually large relative volume change, $\Delta \mathrm{V} / \mathrm{V}$, which can be as large as $20 \%$. In the liquid there is a strong competition between screening and steric (excluded volume) effects which leads to the very characteristic feature of charge ordering, a regular alternation of oppositely charged neighbor shells around a central ion $(35)$. This leads to an oscillatory behaviour of the pair disttribution functions $g_{\alpha \beta}(\Omega)\left(\alpha_{1} \beta= \pm\right)$, which also occurs in concentrates solutions of electrolytes.

Molten salts undergo a liquid-gas transition which terminates at a critical point corresponding roughly to $\gamma \cong 10$ and $\eta \simeq 0.1$ (36). This phase transition is particularly interesting because it is accompanied by a kind of "conductor-insulator" (or Mott) transition due to ion pair formation in the low density vapour. While ions are large$1 y$ dissociated in the denser liquid phase, which is essentially coulombic in nature, a correct Statistical Mechanics description of the dilute vapoux phase must take ion pairs (forming dipolar molecules), and possibly larger clusters into account. Failure to do so has led to highly erroneous estimates of the critical parameters in the framework of the RPM of eq.(2I)(37). In particular, the familiar "mean spherical approximation" (MSA) leads to a critical density $\eta_{c} \simeq 0.01$, which is too low by an order of magnitude.

To obtain a correct description of the low density vapour and of liquid gas coexistence, Bjerrum's(38) classic "ion pairing" idea has recentIy been revived (39). In its crudest form this theory is based on the observation that the integrand of the partition function for a pair of oppositely charged ions, i.e.:

$$
4 \pi \exp \left\{-v_{+-}(r) / k_{B} T\right\} r^{2}=4 \pi e^{b / r} r^{2}
$$

has a minimum at $r=b / 2$, i.e. at one half the Bjerrum length; this distance defines an ion pair as one whose separation is less than b/2, as long as $b / 2>\sigma$. The restriction of the pair size, i.e. of the available confjguration space, leads in fact to an upper bound to the free energy 395 . The system is then considered as a gas of free ions, which are treated by Debye-Hückel theory, and of ion pairs which behave as an ideal (non-interacting solute). The fraction of ions that are paired is finally obtained by minimizing the total free energy, which immediately yields the equilibrium constant for the ion pairing equilibrium. A recent extension of this idea shows that the fraction of un-paired ions is negligible compared to those engaged in pairs and larger neutral clusters(39).

In the highly dissymmetric case of macroions in the presence of counterions and stray ions, the mechanism similar to ion pairing in symmetrical electrolytes is the formation of Stern layers and the phenomenon of. "ion condensation" which will be briefly examined in the following 
section.

5. THE TWO-DIMENSIONAL COULOMB GAS

The symmetrical two-dimensional Coulomb gas is a system of oppositely charged hard disks interacting via the $2 \mathrm{~d}$ Coulomb potential, and is hence the exact counterpart of the $3 \mathrm{~d}$ RPM of eq.(2I); setting $\varepsilon=1$, the $2 \mathrm{~d}$ potential reads:

$$
\begin{aligned}
v_{\alpha \beta}(\lambda) & =\infty ; r<\sigma \\
& =-z_{\alpha} z_{\beta} e^{2} \ln (r / L) ; r>\sigma
\end{aligned}
$$

A very interesting feature is that, contrarily to the $3 \mathrm{~d}$ case, a system of point charges $(\sigma=0)$ will be stable as long as

$$
T>e^{2} / 2 k_{B} \text {, or } \Gamma<2 \text {. }
$$

The partition function will be finite under this condition, since the contribution of a pair of oppositely charged particles will then remain integrable at vanishing distances.

$$
\begin{gathered}
\int_{0}^{R} \exp \left\{-v_{+}(r) / k_{B} T\right\} r d r=\int_{0}^{R} \exp \{-\Gamma \ln (r / L)\} r d r \\
=L^{\Gamma} \int_{0}^{R} r^{1-\Gamma} d r<\infty \quad \text { if } \Gamma<2
\end{gathered}
$$

At $\Gamma=2$, the internal energy and the specific heat diverge and the plasma of $\mathrm{N}$ positive and $\mathrm{N}$ negative ions recombines into an ideal gas of $N$ "collapsed" pairs. If $\rho=2 \mathrm{~N} / \mathrm{S}$ is the total areal density, the equation-of-state for $\Gamma<2$ is the same as that of the $2 d$ OCP (eq. (20)), while for $\Gamma>2$ it reduces to that of an ideal gas $(31)$ :

$$
\begin{aligned}
\frac{\beta P}{\rho} & =1-\Gamma / 4 & & ; \Gamma<2 \\
& =1 / 2 & & ; \Gamma>2
\end{aligned}
$$

In the dissymmetric case of $\mathrm{N}$ ions of charge $+\mathrm{Ze}$ and $\mathrm{N} \mathrm{Z}$ ions of charge -e the plasma will be stable as long as $T>\mathrm{e}^{2} /\left(\mathrm{k}_{\mathrm{B}} 2 \mathrm{Z}\right)$, and there will be $Z$ successive "recombinations", with as many breaks in the equation-of-state, which now reads $(40)$ :

$$
\begin{aligned}
& \frac{\beta P}{\Gamma}=\frac{z+1-z}{z+1}\left[1-\frac{\Gamma}{4}(z-z)\right] ; \\
& \frac{2}{z+1+z}<\Gamma=\frac{e^{2}}{k_{\beta} T}<\frac{2}{z-z} ; 0 \leqslant z \leqslant z
\end{aligned}
$$

The analogy between these "collapse transitions" in the $2 \mathrm{~d}$ coulomb gas and Manning "s(41) "ion condensation" on linear polyelectrolytes is striking. According to Manning's picture monovalent counterions will "condense" on a linear polyelectrolyte chain when the ratio of the Bjerrum length $b$ over the spacing a between monovalent monomers on the chain will exceed the critical ratio $\xi=1$. For ions of finite size $(\sigma \neq 0)$, the collapse transition goes over into a transition between a high temperature Coulomb gas, and a low temperature dipolar gas in which oppositely charged ions are paired into neutral "molecules", very much as in the $3 \mathrm{~d}$ case discussed in the preceding section(27). Although the existence of such a "conductor-insulator" transition in $2 \mathrm{~d}$ has been rigorously established (42), the 
precise phase diagram is not yet known quantitatively. Calculations along Bjerrum old ideas (38) are presently under way. Ion pairing plays also a central role in the case of point ions $(\sigma=0)$, in the range $1<\Gamma<2$, where it strongly influences pair correlations $(42,40)$.

6. "LIQUID-GAS" TRANSITION IN CHARGED COLLOIDAL SUSPENSIONS

It is we11 known that for sufficiently strong screening (i.e. in the presence of a sufficient amount of added salt), the electrostatic repulsion (1) between double-layers becomes short-range and "uncover" the van der Wals attraction between colloidal particles. This attraction is ultimately responsible for the coagulation of the colloid(2). The total potential between two colloidal particles is then of the form:

$$
v(x)=J \frac{e}{x}^{-K(x-1)}-\frac{A}{12} h(x)
$$

where $x=r / \sigma$ is the reduced distance between the centers of two particles $K=K \sigma$ is the reduced inverse Debye length, $\mathrm{J}=\gamma^{2} / \sigma$ is the electrostatic coupling constant, $A$ is Hamaker's constant and the function $h(x)$ describes the van der Waals attraction between colloidal spheres:

$$
\begin{array}{rlrl}
h(x) & =\frac{1}{x^{2}-1}+\frac{1}{x^{2}}+2 \ln \left(1-\frac{1}{x^{2}}\right) \\
& =\frac{1}{3 x^{6}} & ; x & \gg 1 \\
& \simeq \frac{1}{2(x-1)} & ; x \simeq 1
\end{array}
$$

Whenever the ratio $\xi=24 J / K A$ exceeds 2.7 , the potential (27) exhibits a positive maximum at $x=x_{M}>1$ and a subsequent minimum at $x=x_{m}$ : Coagulation will be prevented as soon as the Coulomb barrier $v\left(x_{M}\right)^{m}$ is substantialiy larger than the thermal energy $k_{R} T$, and the physical diameter $\sigma$ of the particles can be safely replaced by the effective diameter $\sigma_{\text {eff }}=\sigma x_{M}$. If the secondary minimum is sufficiently deep $\left(\left|v\left(x_{m}\right)\right| \gtrsim k_{B} T\right)$ there exists then the possibility that chargestabilized dispersions of colloidal particles will undergo a reversible phase separation into a dilute "gas" phase coexisting with a concentrated "liquid" phase, under the combined action of the screened electrostatic repulsion and the van der Wals attraction. The analogy with the diagram of simple molecular substance would then be complete, with the colloidal dispersion exhibiting the usual gas, liquid and solid phases. This possibility has recently been explored within the framework of the thermodynamic perturbation theory of simple liquid $(18)$ by Grimson ${ }^{43)}$ and Victor and Hansen (44). The first of these papers applies only to weakly charged microemulsions, while the second considers the case of highly charged spherical colloids of arbitrary size. The latter work determined the range of physical parameters $(\mathrm{J}, \mathrm{A}, \mathrm{K})$ over which the predicted phase separation may be observable experimentally in aqueous solutions(44). The conclusions of this work are the following. A "liquid-gas" transition should indeed be observable at physically acceptable temperatures $(\mathrm{T}>273 \mathrm{~K}$ ), provided the values of the electrostatic and Hamaker coupling constants lie above a critical curve shown in fig.1. In that range the temperature $\mathrm{T}$ and packing fraction $\eta_{c}$ of the liquid-gas critical point increases wifh increasing salt concentration (i.e.K), but the two-phase system will be stable against coagulation only over a very limited range of salt concentrations. 


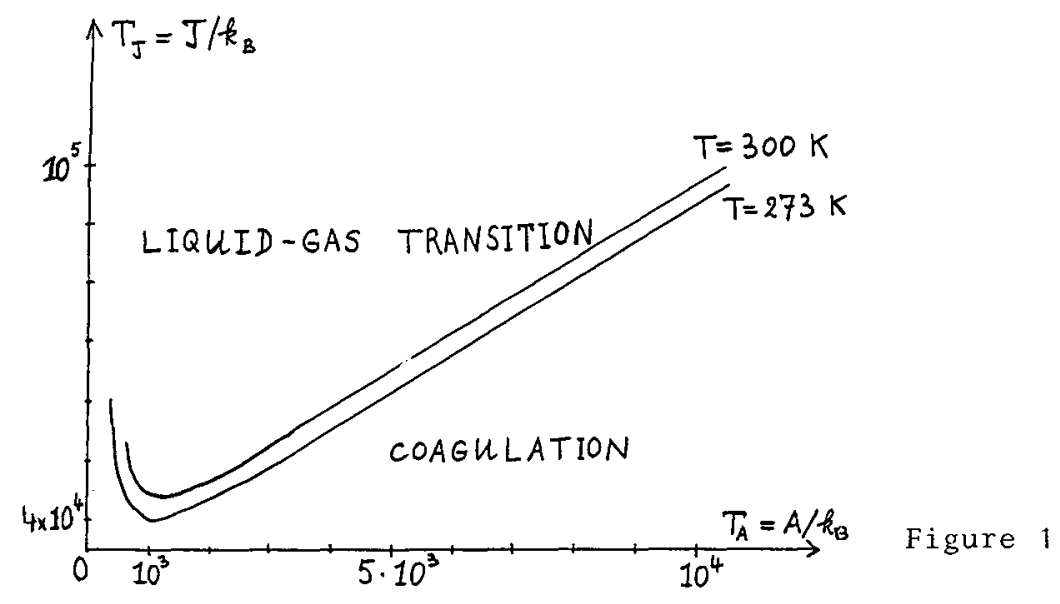

Typically these conditions can be met, for reasonable values of Hamaker's constant ( $A \simeq 2.5 \times 10^{-20}$ Joule) and of the surface potential $\left(\psi_{\mathrm{O}} \simeq 25 \mathrm{mV}\right)$, if the diameter of the colloidal particles is $\sigma \bumpeq 0,5 \mu$ or Iarger, and for salt concentrations of the order of $0.01 \mathrm{M}$ (then

$K=K \sigma>100$ ). The resulting 1 iquid gas coexistence and spinodal curves look very much like those typical of simple molecular fluids. Un.der very favourable conditions a gas-liquid-solid triple point should be observable, but under the conditions of strong screening necessary for such a coexistence, the repulsion is sufficiently steep that an FCC, rather than a BCC colloidal crystal would be the more stable structure. It is speculated that with increasing salt concentration a transition from $B C C$ to FCC should be observable in the solid phase.

\section{REFERENCES}

1. M. Baus and J.P. Hansen, J.Stat.Phys.31 (1983) 409

2. E.J.W. Verwey and J.T.G. Overbeek: "Theory of the Stability of Lyopholic Colloids" (Elsevier, Amst. 1948).

3. G.M. Be11, S. Levine and L.N. McCartney, J.Coll. Interf.Sc.33 (1970) 335

4. M. Baus and J.P. Hansen, Phys.Rep.59 (1980)1

5. F.H. Stilinger and R. Lovett, J. Chem.Phys.49 (1968) 1991

6. J.C. Brown, P.N. Pusey, J.W. Goodwin and R.H. Ottenvil1, J.Phys.A 8 (1975)664.

7. F. Grüner and W. Lehmann, J. Phys.A 13 (1980) 2155

8. M.J. Grimson, J. Chem.Phys.79 (1983) 5070

9. E.L. Pollock and J.P. Hansen, Phys.Rev.A 8 (1973) 3110 .

10. H. Minoo, C. Deutch and J.P. Hansen, J. de Phys.Lettres $\underline{38}$ (1977)L 191

11. G. Pastore and M.P. Tosi, to be published

12. W.L. Slattery, G.D. Doolen and H.E. De Witt, Phys.Rev.A 26 (1982) 2255 .

13. J.D. Weeks, Phys.Rev.B 24 (1981) 1530

14. M.J. Gillan, J.Phys. C7 (1974) L 1

15. S. Galam and J.P. Hansen, Phys.Rev. A 14 (1976) 816

16. J.P. Hansen, J. Phys. C 14 (1981) L $15 \overline{1}$

17. K.K. Mon, R. Gann and D. Stroud, Phys.Rev.A 24(1981) 2145

18. J.P. Hansen and I.R. McDonald, "Theory of Simple Liquids" (Academic Press, London, 1976)

19. J.P. Hansen and J.B. Hayter, Mol.Phys.46 (1982) 651.

20. R. Evans and Sluckin, J. Phys. C 14 (1981) 3137 
21. P. Pieranski, Contemp.Phys. 24 (1983) 25

22. C.C. Grimes and G. Adams, Phys.Rev.Letters 42(1979)795

23. F.I.B. Williams, Surf.Sci. 113 (1982) 371

24. R.C. Gann, S. Chakravarty and G.V. Chester, Phys.Rev.B20(1979) 326

R.K. Kalia, P. Vashista and S.W. de Leeuw,Phys.Rev.B23(1981)4794

25. B.J. Alder and T.E. Wainwright, Phys.Rev.127 (1962) 359,A.F.Bakker, C. Bruin and H.J. Hilhorst, Phys.Rev.Letters 52 (1984) 449

26. J.M. Kosterlitz and D.J. Thouless, J. Phys. C $6(19 \overline{73}) 1181$,

D.R. Nelson and B.Halperin, Phys.Rev.B 19 (1979) 2457

27. R.H. Morf, Phys.Rev.Letters 43 (1979) $9 \overline{3} \overline{1}$

28. P. Pieranski, Phys. Rev.Letters 45 (1980) 569

29. R.K. Kalia and P. Vashishta, J. Phys.C 14 (1981) L 643

30. E.H. Hauge and P.C. Hemmer, Phys.Norv.5 (1971) 209

31. J.M. Caillo1, D. Levesque, J.J. Weis and J.P. Hansen, J.Stat. Phys. 28 (1983) 325

32. S.W. de Leeuw and J.W. Perram Physica 113A (1982) 546.

33. Ph. Choquard and J. Clerouin, Phys.Rev.letters 50 (1983) 2086

34. For a review, see J.E. Enderby and G.W. Neilson, Adv. in Physics 25 (1980) 323

35. H.L. Friedman and B. Larsen, J. Chem.Phys.70 (1979) 92

36. G.R. Stel1, K.C. Wu and B. Larsen, Phys.Rev.Letters 37 (1976) 1369

37. N. Bjerrum, Kg1.Danske Vidensk, Se1skab $\underline{7}$ (1926) no.9

38. M.J. Gillan, Mo1.Phys.41 (1980) 75

M.J. Gillan, AERE Harwel1 preprint T.P. 913 (1981)

39. J.P. Hansen and P. Viot - to be published

40. G.S. Manning, J. Chem. Phys.51 (1969) 924

41. J.P. Hansen and P. Viot, Physics Letters 95 A (1983) 155

42. M.J. Grimson, J. Chem.Soc. Faraday Trans, $2,79(1983) 817$

43. J.M. Victor and J.P. Hansen, to appear in J.de Physique, Letters (1984). 